\title{
RETOS DE LA FORMACIÓN EN PSICOLOGÍA EN LA UNIVERSIDAD DE COSTA RICA FRENTE A LOS POSIBLES ESCENARIOS FUTUROS
}

\section{CHALLENGES IN PSYCHOLOGY INSTRUCTION AT THE UNIVERSITY OF COSTA RICA IN FACE OF POSSIBLE FUTURE SCENARIOS}

\author{
Zaida Salazar Mora ${ }^{1}$ \\ zaidasalazar@hotmail.com \\ Adriana Maroto Vargas ${ }^{2}$ \\ adriana.maroto.vargas@gmail.com \\ Jorge Esteban Prado-Calderón ${ }^{3}$ \\ jorgepradopsi@gmail.com
}

Fecha de recepción: 6 enero 2014 - Fecha de aceptación: 27 julio 2014

\begin{abstract}
Resumen
En la actualidad, el avance del modelo neoliberal presenta retos que la Universidad Pública debe enfrentar. Estos ameritan reflexionar sobre los futuros escenarios de la sociedad y el rol que deberán desempeñar las y los profesionales en Psicología. En este artículo se presentan las principales conclusiones de las entrevistas llevadas a cabo con cuatro profesionales en Psicología de amplia trayectoria y diversas perspectivas teóricas sobre los posibles escenarios, retos y competencias que deberá enfrentar la disciplina. Las conclusiones destacan el impacto de las tecnologías de la comunicación en la sociedad futura, así como los retos de la Psicología en cuanto a formar profesionales con conciencia social, pensamiento crítico y habilidades para desarrollar modalidades de intervención más comunitarias. Asimismo, se evidencia la importancia del desarrollo de la capacidad para trabajar en equipos interdisciplinarios, la cual ha sido dejada de lado en los programas de formación. Finalmente, se reflexiona sobre el papel que la Universidad Pública debe jugar para la consecución de una Psicología costarricense que propicie la transformación que la sociedad necesita para alcanzar un adecuado abordaje de la salud mental y el bienestar.

Palabras clave: Psicología, competencias, enseñanza superior, Universidad Pública, cambio tecnológico, interdisciplinariedad, psicología comunitaria, pensamiento crítico, conciencia social, salud mental.
\end{abstract}

\begin{abstract}
Nowadays, the advance of the neoliberal model brings challenges that Public Universities must face. It is necessary to discuss about future scenarios of our society, and the role that Psychology professionals should play. This paper shows the main findings from the interviews to four experience psychologists who have diverse theoretical perspectives about the possible scenarios, challenges and competences that
\end{abstract}

1 Docente Jubilada de la Escuela de Psicología, de la Universidad de Costa Rica.

2 Docente de la Escuela de Psicología, de la Universidad de Costa Rica.

3 Docente de la Escuela de Psicología, de la Universidad de Costa Rica. 
the discipline must address. The findings highlight the potential impact of communication technologies on future society, as well as the discipline's challenges in instructing professionals with social awareness, critical thinking and the skills to develop community intervention modalities. It also evidences the importance of developing the ability to work in interdisciplinary teams which has been neglected in the training programs. Finally, it debates on the role that Public Universities must play in attaining the Costa Rican Psychology that promotes the transformation required by society in order to achieve an adequate approach to mental health and wellness.

Key words: Psychology, competences, higher education, Public University, technological change, interdisciplinarity, community psychology, critical thinking, social awareness, mental health.

\section{Introducción}

\section{Retos de la formación en Psicología en Costa Rica ante los posibles escenarios futuros}

El presente trabajo surge de la inquietud de discutir sobre las perspectivas y retos en la formación de profesionales en Psicología desde la Universidad Pública y, particularmente, desde la Universidad de Costa Rica (UCR). Para esto, se realizaron entrevistas semiestructuradas a profesionales seleccionados bajo cuatro criterios, a saber, tener el grado académico de doctorado, amplia experiencia académica y profesional, diversidad en cuanto a campos de inserción y enfoques teóricos y paradigmáticos, así como tener la perspectiva de hombres y mujeres. De ninguna manera se pretende agotar con estas entrevistas -y con el artículo mismo- la discusión ni buscar ningún nivel de representatividad en las personas entrevistadas. Al contrario, se pretende generar insumos que permitan abrir el debate, el cual se considera parte fundamental en el proceso de evaluación continua que debe realizar cada carrera.

En este artículo se exponen las principales reflexiones generadas a partir del análisis de los diálogos mantenidos con el Dr. Henning Jensen Pennington, la Dra. Mirta González Suárez, el Dr. Mauricio Leandro Rojas y la Dra. Mónica Salazar Villanea, profesionales con amplia experiencia en docencia e investigación, quienes han desarrollado gran parte de su carrera académica en la Escuela de Psicología de la UCR.

Las entrevistas, realizadas individualmente, tuvieron una duración aproximada de una hora y fueron grabadas y transcritas para su posterior análisis. Se implementó el formato oral de consentimiento informado, por medio del cual se verificó la participación voluntaria y sin coerción y se solicitó la autorización de las personas participantes para ser citadas en el informe final.

Cabe resaltar que durante la entrevista se solicitó a cada persona que definiera los escenarios futuros posibles que visualiza en los próximos 20 años, así como los retos que cada uno de estos implicaría para la formación de profesionales en Psicología. Por lo tanto, cada quien, desde su bagaje teórico y su área de interés, definió las prioridades sobre las cuales se centró la entrevista. De esta manera, se produjo un material amplio respecto a las temáticas abordadas. Ante esto, se tomó la decisión de centrar el artículo en aquellos ejes que fueron identificados como comunes en las entrevistas. Existe claridad que esta decisión significó excluir material sumamente valioso, sin embargo, su inclusión generaría una gran dispersión en el análisis.

El artículo se estructuró con base en dos apartados. En el primero de ellos, se presentan algunas reflexiones sobre el papel de la Universidad Pública en el contexto nacional, aspecto que se consideró importante para ubicar el análisis y la discusión que se está planteando. En un segundo apartado se exponen los principales ejes comunes identificados en las entrevistas, incluyendo los escenarios futuros y los retos, así como su vinculación analítica con el quehacer de la Escuela de Psicología. 


\section{El papel de la Universidad Pública en la Costa Rica del siglo XXI}

En un momento histórico en que el neoliberalismo se ha consolidado como régimen social y económico en las sociedades latinoamericanas en general y la costarricense en particular, adquiere una gran pertinencia la discusión sobre el papel de la Universidad Pública en esta coyuntura, con el fin de enmarcar el actual contexto de los retos de la formación de profesionales en Psicología.

Según Boaventura de Sousa Santos (2005), para que exista la Universidad deben estar plenamente desarrolladas en la institución la docencia (grado y posgrado), la investigación y la acción social. Afirma este autor que ante la ausencia de una de ellas existirá educación superior, mas no Universidad. Por su parte, la Asociación de Unidades Académicas de Psicología (AUAPSI, 2012) ha señalado que los pilares fundamentales de la Universidad Pública son la autonomía, la gratuidad, la laicidad y la vinculación de la institución con el entorno social en que se inserta, sin embargo, actualmente estos pilares están cuestionados por el modelo neoliberal.

Históricamente la UCR ha jugado un papel fundamental en los procesos de transformación social tendientes a la construcción de modelos sociales más justos en nuestro país. No obstante, no es un secreto que a lo interno de la misma institución existen posturas ideológicas en disputa, por ejemplo respecto al contenido ético y político de la acción social, así como la pertinencia y las implicaciones de la vinculación externa remunerada a partir de proyectos de investigación y acción social.

Según Boaventura de Sousa Santos (2005), la Universidad Pública ha sido y es una de las muy pocas instituciones que establecen una correlación entre el presente y el futuro a mediano y largo plazo, a través de procesos planificados en la docencia, la investigación y la acción social. Sin embargo, más que los aspectos académicos, científicos, éticos y políticos, en muchos casos la lógica administrativa y burocrática de la Universidad adquiere mayor peso en la planeación y ejecución de los proyectos que se impulsan desde la institución.

Por su parte, Meoño (2011) enfatiza que la Universidad es una institución en disputa ante la lógica neoliberal que pretende convertirla en una mercancía, lo cual se evidencia en las condiciones que se le imponen para que sea competitiva en el mercado y cumpla su papel en la formación ideológica de nuevas generaciones, como fuerza reproductiva al servicio de la reproducción y valorización del capital y el sistema como tal.

Coincidente con esta línea, Boaventura de Sousa Santos (2005) afirma que la autonomía pedagógica y científica de la Universidad está en crisis por depender del financiamiento estatal. Este tema adquiere gran vigencia en nuestro país en el marco de la ratificación del préstamo con el Banco Mundial y las negociaciones entre las Universidades Públicas con el gobierno en torno al Fondo Especial para el Financiamiento de la Educación Superior Estatal (FEES).

En un Estado costarricense dirigido por gobiernos neoliberales en que las políticas públicas y el financiamiento dirigido al sector social no son prioridad, la Universidad Pública ha enfrentado serias amenazas respecto a su financiamiento, especialmente en carreras que no responden a esta lógica mercantilista y más bien se convierten en cuestionadoras del sistema. Claros ejemplos de esto son la no aprobación de nuevas promociones de la carrera de Psicología en la Sede de Limón desde el año 2012 y el acuerdo del Consejo de Sede para no abrir una nueva promoción de esta misma carrera en la Sede de Occidente para el año 2013, hecho este último que no se concretó gracias a la movilización estudiantil.

Según Henning Jensen (comunicación personal, 19 de julio, 2012), en el proceso de acentuación de la globalización se van a ir involucrando aspectos que habían estado un poco al margen y particularmente se refiere a la educación pública en todos sus niveles. Por lo tanto, considera que a estas instituciones se les exigirá nuevas formas de legitimación y posicionamiento frente a la realidad nacional, aspectos que han sido parte de la discusión en las negociaciones referentes al financiamiento para los próximos años.

Al respecto, Mauricio Leandro (comunicación personal, 18 de julio, 2012) afirma que a pesar de las amenazas existentes, no cree posible que en un futuro cercano desaparezca en el país la Universidad 
Pública, sin embargo considera que no estarán ausentes las luchas y los procesos de movilización por parte de estudiantes y docentes, así como de la sociedad en general, ante estos embates.

Por lo tanto, queda claro que el proceso de formación de nuevas y nuevos profesionales se enmarca en procesos de tensión respecto al modelo de Universidad Pública según se ha concebido históricamente en la región latinoamericana y las amenazas que se enfrentan desde la mercantilización de la educación superior en el país.

\section{Desarrollo}

\section{Posibles escenarios sociales a futuro}

En el marco de este proceso de globalización y fortalecimiento del neoliberalismo, las entrevistas permitieron indagar sobre los escenarios posibles, tanto a nivel internacional como nacional en el marco de los próximos 20 años. Esto con el objetivo de discutir los retos que se enfrentarán para la formación de profesionales en Psicología. Como se mencionó anteriormente, debido a la diversidad de paradigmas e inserciones profesionales de cada una de las personas entrevistadas, estas enfatizaron en aspectos diversos, los cuales se introducen a continuación para posteriormente centrar el foco de atención en un punto común en que convergieron todas ellas.

Para Henning Jensen los procesos de globalización van a impactar en las formas de participación ciudadana ante la deslegitimación que enfrentan las estructuras gobernantes.

La sociedad como un todo se enrumba hacia una mayor participación de la ciudadanía en asuntos de interés global para una sociedad, pero eso no va a suceder sin tensiones porque todavía hay estructuras que ofrecen resistencia a esa mayor participación (comunicación personal, 19 de julio, 2012).

En el caso de Mauricio Leandro el reto identificado es cómo construir ciudad y cómo impulsar un desarrollo urbano sostenible que promueva la vida activa. Para él es preocupante el poco interés que hay por lo público.

En este momento lo público incluso en algunos casos es una mala palabra (...) Lo que pasa es que cuando uno dice "lo público", está hablando también de espacio público. El espacio público se pierde. Y no pareciera que haya un esfuerzo coordinado de las instituciones o del Estado de recuperar lo público, los bienes comunes (comunicación personal, 18 de julio, 2012).

Por su parte, para Mónica Salazar uno de los cambios globales más importantes tendrá que ver con el envejecimiento demográfico. Este constituye un reto para el cual Europa se fue preparando de manera paulatina, aunque en el caso de América Latina hasta el momento no ha existido una preparación similar.

Finalmente Mirta González destacó aspectos diversos, siendo uno de ellos el tema de los derechos humanos en general y los derechos laborales en particular. Respecto a este último tema llamó la atención sobre los cambios en el lenguaje que lejos de ser inocentes responden a la lógica neoliberal. Enfatizó que actualmente se llama "colaboradoras" a las personas trabajadoras, lo cual conlleva serias implicaciones.

Todos los días se cuestionan por parte de las grandes empresas los derechos laborales. El objetivo (...) es bastante profundo y maquiavélico, porque se cambian las palabras, por ejemplo el uso del término "colaboradora" en lugar de "trabajadora". Si nada más "colaborás", se pierde la memoria histórica de las luchas por los derechos laborales. Solo falta que digan que se trabaja por "voluntariado" para encubrir la relación entre las personas asalariadas y sus patronos o patronas. Otra forma de no reconocer los logros es denominar a los derechos laborales (aguinaldo, cesantía, salario mínimo, montos por enfermedad, licencia de maternidad, etc.) como "cargas sociales", esto en el marco de la "competitividad" y esta última palabra implica bajar los costos de las empresas al mínimo. ¿Cómo que cargas sociales? Se trata de condiciones justas, pero todo aquello que disminuya la alta ganancia se presenta como un problema a erradicar (comunicación personal, 2 de noviembre, 2012). 
Si bien cada uno de estos criterios, desde una primera lectura, parece referir a aspectos muy diversos, desde un análisis un poco más detenido se pueden encontrar ejes comunes para el análisis si se utiliza como punto de partida los impactos del neoliberalismo y la globalización en nuestra vida cotidiana. No obstante, el análisis del artículo estará enfocado en un tema en el cual, a pesar de la diversidad de focos de atención de cada una, coincidieron estas cuatro personas en sus planteamientos. Este tema fue el impacto que están teniendo las nuevas tecnologías de la información y la comunicación (TIC) en la subjetividad y las relaciones interpersonales, tema que no puede pasar inadvertido por la Psicología.

Henning Jensen destaca el papel que está jugando Internet en la manera en que procesamos información. Menciona que el Internet modifica no solo la forma en que juzgamos la información proveniente de la prensa, sino incluso la información cotidiana de nuestro medio. De esta manera, enfatiza en que se está dando una inmediatez de la información pero, principalmente, una pérdida de criticidad, de manera que la información se da por cierta inmediatamente, sin que existan interrogantes sobre la validez de la fuente.

Es en este contexto en el que dentro de la formación profesionalizante en la carrera de Psicología se vuelve urgente, según Henning Jensen, retomar los procesos psicológicos básicos, incluyendo la atención, la percepción, la memoria, el razonamiento, el lenguaje, la comunicación y la emoción, entre otros. Sin duda este es un tema que nuevamente ha venido adquiriendo relevancia y por esa razón, desde el año 2010, el currículo de la Escuela de Psicología en la UCR incluyó, en el primer año, el curso Procesos Psicológicos Básicos, con el cual se busca analizar los modelos teóricos surgidos a partir de la investigación de cada proceso psicológico básico, así como de sus interacciones.

Mauricio Leandro y Mirta González coinciden en que las nuevas TIC están provocando una digitalización o virtualización de las relaciones interpersonales, la cual no tiene el mismo impacto que la comunicación personal, ya que, por ejemplo, se pierde en gran parte el componente de comunicación no verbal.

Sin embargo, Mónica Salazar y Mirta González advierten que este fenómeno tiene sus implicaciones positivas. El gran riesgo consiste en que las relaciones virtuales vengan a suplantar por completo los contactos y las relaciones interpersonales, no obstante en las nuevas TIC existe un gran potencial para hacer denuncias públicas de hechos de corrupción, para tener acceso a información de interés público y para brindar mayores oportunidades de vinculación a personas que por alguna discapacidad tiene dificultades para movilizarse. A esto Mónica Salazar añade que existen estudios enfocados en diferentes etapas de la vida, sobre todo en la adolescencia, que abordan la posibilidad de jugar a tener identidades virtuales y los efectos de las TIC sobre el desarrollo de la identidad.

La relación del ser humano con la tecnología es compleja, y la configuración de la sociedad y la cultura juega un papel fundamental en la misma. La investigación señala influencias positivas y negativas de la tecnología sobre la socialización, siendo este uno de los grandes problemas y retos de la investigación psicológica para el cual se debe tomar perspectiva a fin de comprender y juzgar este fenómeno, considerando el contexto histórico. En la actual sociedad de la información se dan nuevas formas de comunicación donde, según Adell (1997), "el espacio y el tiempo ya no son condicionantes de la interacción social, del mismo modo que las fronteras y los límites nacionales no representan barreras para la circulación del capital, de la información, de los mercados" (párr. 39). Este autor cita como ejemplo a las comunidades virtuales donde grupos de personas, dispersos y temporalmente no sincronizados, comparten un interés y utilizan las redes informáticas como una vía de comunicación cómoda y de bajo costo. Esta "interactividad" junto con la "deslocalización" que definen las nuevas TIC posee implicaciones cruciales en todos los ámbitos de la experiencia humana. Así, se habla de la netgeneration con una nueva dimensión comunicativa ampliando considerablemente el campo social (Adell, 1997). 
No obstante, respecto a este tema y desde un punto de vista crítico, debe tenerse muy presente que las nuevas TIC aún no tienen un alcance universal, e incluso se afirma que la brecha digital tiene una estrecha relación con la desigualdad social (Programa Sociedad de la Información y el Conocimiento [PROSIC], 2006).

Entre los factores causantes de la brecha, queda claro que las tecnologías no constituyen un elemento diferenciador en sí mismas, por lo que su origen se encuentra en las disparidades sociales de carácter estructural como la desigual distribución del ingreso, en la dotación selectiva de la infraestructura de comunicaciones y en el desigual nivel educativo de personas y comunidades (p. 221).

Esto queda en evidencia cuando se conocen los niveles de conectividad a Internet por cantón en nuestro país, en donde aquellos ubicados en el Área Metropolitana y con mayores niveles de desarrollo económico y social, tales como Montes de Oca, Escazú, Curridabat y Belén, encabezan la lista, mientras que los ubicados en las zonas periféricas, tales como Matina, Upala y La Cruz, se encuentran en la parte más baja del ranking (Fernández, 2013).

Por lo tanto, la investigación sobre el este tema no debe desconocer la desigualdad social y el acceso a recursos, entre los cuales se pueden incluir las nuevas TIC.

Respecto a la formación profesional, Henning Jensen destaca que el acceso a Internet abre toda una ventana para la actualización permanente y el establecimiento de relaciones con profesionales y universidades de todas partes del mundo. En términos institucionales, la Universidad no puede quedarse aislada y debe integrarse cada vez más a redes nacionales e internacionales de cooperación que permitan potenciar todos los recursos (económicos, humanos y tecnológicos) para el avance del conocimiento en general y de la Psicología en particular.

La globalización tiene que ver con la creación cada vez más acentuada de redes de cooperación internacionales, que en el caso de la educación superior pública puede ser un escenario más bien muy positivo en tanto que se construyen lazos de solidaridad (Henning Jensen, comunicación personal, 19 de julio, 2012).

A nivel institucional, la UCR ha dado gran importancia al tema de las TIC en los últimos años, al punto de que para el 2007 se contaba con cerca de 17 programas, proyectos, acciones y actividades permanentes de integración de las TIC en la academia (PROSIC, 2008). Además de la creación del portal institucional y sitios Web, desde el 2005 la UCR cuenta con el PROSIC (www.prosic.ucr.ac.cr) y desde el 2011 con el Centro de Investigaciones en Tecnologías de la Información y Comunicación (www.citic. ucr.ac.cr). Asimismo, dentro de la institución se ha promovido y capacitado en la implementación de las TIC en la docencia desde febrero de 2006, cuando entra en funcionamiento la Unidad de Apoyo a la Docencia Mediada con Tecnologías de la Información y la Comunicación (METICS, portafoliovirtual. ucr.ac.cr). Todos estos esfuerzos ayudaron a que en noviembre de 2009 se aprobara la implementación del portal de aulas virtuales de la UCR, conocido como Mediación Virtual (mediacionvirtual.ucr.ac.cr).

Adicionalmente, la UCR cuenta con múltiples recursos en línea tales como bases de datos de texto completo, su propio portal de revistas académicas (revistas.ucr.ac.cr) y repositorios de investigaciones (kerwa.ucr.ac.cr) y trabajos finales de graduación (repositorio.sibdi.ucr.ac.cr:8080/jspui), a lo que se añade que desde octubre de 2012 se encuentra inscrita en RedCLARA, una red avanzada de investigación científica y académica en línea que facilita las redes de trabajo internacionales, teleconferencias, entre otros.

En el caso de la Escuela de Psicología, esta cuenta con un objetivo explícito con el que busca promover el uso de las TIC en la docencia. A raíz de lo anterior, se ha trabajado en conjunto con las instancias de la universidad mencionadas y todos los años se divulgan decenas de actividades de capacitación en temas como e-learning, aprendizaje colaborativo en línea y el uso de herramientas tecnológicas para la docencia tales como las redes sociales, las herramientas de Google y aplicaciones específicas como Prezi, CmapTools, etc. En la actualidad cerca de 14 grupos de la Escuela utilizan la plataforma 
de Mediación Virtual y en muchos otros se implementan recursos tecnológicos tales como Dropbox, Youtube, Blogger, etc. Adicionalmente, se cuenta con una revista electrónica (Wímb Lu) y se utiliza la plataforma Lime Survey para recolectar datos en varios trabajos finales de graduación (Chaves, Ramos \& Sánchez, 2013; Hernández \& Ulloa, 2014). Sin embargo, a futuro será necesario aumentar el uso fundamentado de las TIC para la docencia con medidas tales como la capacitación didáctica del personal académico, un mayor y más efectivo aprovechamiento de la plataforma de Mediación Virtual, y la implementación de importantes opciones actuales como la edición de videos pedagógicos, las teleconferencias interactivas, los foros para la discusión y construcción colectiva del aprendizaje, y las distintas modalidades de evaluación en línea.

\section{Retos de la Psicología}

Según la AUAPSI (2012), la Psicología ha contribuido de manera importante a las sociedades latinoamericanas durante el siglo XX, sin embargo le corresponde incrementar su protagonismo en el siglo actual. En el caso particular de la Escuela de Psicología de la UCR, esta se ha planteado la misión de "propiciar la transformación de la sociedad mediante la docencia, la investigación y la acción social, con base en la formación de profesionales en Psicología capaces de incorporarse al mercado laboral con preparación científico-académica amplia y flexible, que les permita adaptarse a las circunstancias históricas del país y del mundo" (Salazar, 2009).

En congruencia con la misión propuesta, las personas entrevistadas coinciden en que uno de los principales retos de la Psicología es mantenerse como una disciplina crítica y cuestionadora del orden social. La conciencia social y pensamiento crítico ha sido una de las distinciones de las y los estudiantes de Psicología de la UCR y mantiene total vigencia. Se requiere el fomento y desarrollo de una capacidad para entender los problemas sociales y abordarlos críticamente.

En ese sentido, la Psicología no puede quedarse en la academia, sino que debe tomar posición pública y clara respecto a temas de relevancia nacional. Como lo menciona Henning Jensen, "hay una cosa que yo creo que nunca acaba, que es la actitud crítica de cara a la sociedad, porque nosotros siempre tenemos que mejorar la sociedad" (comunicación personal, 19 de julio, 2012).

En el escenario de una inserción profesional bajo la lógica neoliberal y la liberalización de la profesión, se advierten ciertos riesgos. Según Mauricio Leandro, la formación profesionalizante permite insertarnos en ese mercado, no obstante el gran reto es el aspecto ético y los espacios de trabajo que gremialmente se empiecen a impulsar. En ese sentido, indica: "lo de las armas es un indicador clarísimo de hacia dónde podría ir caminando este negocio desde la Psicología" (comunicación personal, 18 de julio, 2012).

Con este argumento, Mauricio Leandro hace referencia a la Ley 7530 de Armas y Explosivos en la cual se establecen los exámenes de idoneidad mental, elaborados por profesionales en Psicología, como un requisito para la tenencia y portación de armas de fuego en el país. Esta norma se ha visto gremialmente como una oportunidad de trabajo, sin que existiera o exista actualmente un debate amplio sobre las implicaciones que tiene la portación de armas de fuego en civiles. La calidad de estos exámenes de idoneidad mental ha sido cuestionada ampliamente en la prensa (Arley \& Moya, 2011) y graves irregularidades han sido denunciadas por parte del Colegio Profesional de Psicólogos de Costa Rica (Arley, 2011). No obstante, no ha habido ninguna reforma al sistema establecido en la normativa anteriormente mencionada.

En un sentido similar, Mirta González enfatiza la importancia del pensamiento crítico en la formación de profesionales en Psicología. Menciona que las personas profesionales en este campo deben

\footnotetext{
Tener una cultura general para entender qué es lo que está pasando, cuáles son las fuerzas sociales, cuáles son los derechos humanos, cuáles son los aspectos que están perjudicando los derechos humanos (...) Quien no posea esta visión integral simplemente va a seguir con la línea de manipulación de masas, es decir, va a ser otra persona tan manipulada como todo el resto (...) [Debe] tener mucho conocimiento, conocimiento de la memoria histórica, conocimiento de las fuerzas que hay alrededor, conocimientos de las formas de discriminación que hay, conocimiento incluso legal (Mirta González, comunicación personal, 2 de noviembre, 2012).
} 
Este reto identificado por las personas entrevistadas forma parte de los grandes principios de formación de la carrera de Psicología de la UCR, el primero de los cuales señala el compromiso con la formación de una psicología crítica, solidaria, reflexiva y creativa, que aborde los problemas de la realidad social costarricense, que atienda los desafíos y compromisos planteados y exigidos por la sociedad, y que enriquezca la vida cultural y científica del país y el desarrollo personal de sus habitantes. Asimismo, la visión de la Escuela de Psicología de la UCR enfatiza el proveer una formación humanista, científica y técnica, de cara a la realidad social del país para la construcción de una sociedad solidaria, equitativa, justa y libre, en procura de una vida digna para las personas en el marco del respeto a los derechos humanos (Salazar, 2009).

Una de las diversas formas en que la Escuela de Psicología busca desarrollar en sus estudiantes la capacidad de pensamiento crítico y el compromiso de cara a la realidad nacional consiste en las prácticas profesionalizantes llevadas a cabo dentro de los cursos avanzados. Con estas, todos los semestres cerca de 30 grupos de estudiantes llevan a cabo aproximadamente 250 intervenciones en varias zonas del país. A raíz de lo anterior, se trabaja con cerca de 70 instituciones y se beneficia aproximadamente a 3000 personas todos los semestres. En las prácticas profesionalizantes las y los estudiantes tienen un contacto directo con la realidad nacional e implementan de manera crítica los conocimientos adquiridos durante su formación. Actualmente la carrera ofrece este tipo de prácticas en diversas áreas tales como la psicología social comunitaria, educativa y del desarrollo, clínica y de la salud, y también desde los aportes de la psicobiología.

Por otra parte, un segundo reto de la Psicología costarricense mencionado por las personas entrevistadas se refiere a la necesidad de desarrollar una Psicología que emplee modalidades de intervención más comunitarias y que beneficien a grupos de personas más numerosos.

Mónica Salazar expresa que en un contexto en el que las tarifas mínimas de los servicios en Psicología son evidentemente excluyentes, las y los profesionales de este campo debemos promover la creación de servicios comunitarios. En el mismo sentido, Mirta González enfatiza que las y los profesionales en Psicología deben asumir roles de defensa de los derechos de las personas, y que desde la Psicología se debe promover una salud mental para todos y todas, no solamente para quienes tienen los recursos económicos necesarios para contratar servicios privados. Asimismo, resalta la importancia de las acciones públicas que tengan repercusiones sobre la salud mental:

Debemos tener una formación integral en la cual cada aspecto tenga una visión que yo diría que es hacia la salud mental y hacia el desarrollo conjunto. Parte de la salud mental es poder percibir que lo que nos pasa como personas le pasa a otra gente, que si queremos resolver un problema a veces necesitamos una Política Pública para eso y que no es con un uno, uno, uno que lo vamos a ir resolviendo (comunicación personal, 2 de noviembre, 2012).

Por su parte, Henning Jensen, como se mencionó anteriormente, indica que los cambios de la sociedad actual están modificando la forma en la que procesamos información y el funcionamiento de los procesos psicológicos básicos. En este sentido, afirma que estos cambios deben ser estudiados y que sus implicaciones deben ser tomadas en cuenta en los ámbitos de intervención comunitaria. Asimismo, Mauricio Leandro resalta la importancia de que las y los profesionales en Psicología implementen su capacidad de liderar procesos, y de que estos esfuerzos estén encaminados a la aplicación de la Psicología en la "promoción de salud directamente con las comunidades, no desde instituciones anquilosadas". En sus palabras, se debe concebir al profesional en Psicología "como promotor de salud (...) desarrollando programas en salud comunitaria, salud en las comunidades, en los barrios, desarrollando junto con las Municipalidades y con entes privados, programas de salud" (comunicación personal, 18 de julio, 2012).

Al respecto, Capra (1982/1992, p. 21) nos describe el panorama actual como una profunda crisis mundial: "se trata de una crisis compleja y multidimensional que afecta a todos los aspectos de nuestras vidas: la salud y el sustento, la calidad del medio ambiente y la relación con nuestros semejantes, 
la economía, la política y la tecnología" y además se atreve a afirmar que "la amplitud y la urgencia de la situación no tiene precedentes en la historia de la humanidad". De aquí la necesidad manifestada por las personas entrevistadas de que es fundamental fortalecer las potencialidades participativas y solidarias de diversos sujetos colectivos, en particular las organizaciones de base y las comunidades para enfrentar los problemas que esta crisis presenta. Esta necesidad la señala Armando Campos (s. f.) en su propuesta de un posgrado en Psicología Comunitaria, donde reflexiona que las tareas desplegadas por psicólogas y psicólogos comunitarios se han multiplicado y afianzado, asimismo, sus desarrollos epistemológicos se han organizado en posiciones delineadas con mayor solidez y claridad. Campos considera la psicología comunitaria como una herramienta de desarrollo social y la participación comunitaria como una ruta necesaria para propiciar la integración de las y los marginados y mitigar sus carencias materiales y educacionales.

En esta línea, cabe resaltar que la Escuela de Psicología se ha destacado como una de las unidades académicas que tiene un mayor número de proyectos de acción social en la UCR, tanto en su modalidad de trabajo comunal universitario como de extensión docente. Estos son proyectos comprometidos con situaciones particulares de poblaciones y comunidades de nuestro país; sin embargo, los recursos destinados para la acción social a nivel institucional son bastante limitados y en los últimos años ha existido una tendencia a burocratizar los procesos en aras de establecer mecanismos de control interno, que si bien son fundamentales, dificultan los procesos comunitarios. En este sentido, resulta urgente posicionar la discusión sobre el sentido real de la acción social desde la Universidad Pública, tanto en lo referente al vínculo entre la institución y la sociedad, como en el rol fundamental que esta desempeña en la formación profesionalizante.

\section{Competencias para la formación de profesionales en Psicología}

La formación de profesionales en Psicología, además de la formación en aspectos epistemológicos, teóricos y metodológicos, requiere el fomento del desarrollo de ciertas competencias que les faculten para la inserción profesional. En las entrevistas se destacaron aspectos diversos que se retoman a continuación.

Mónica Salazar y Mauricio Leandro resaltan la importancia de desarrollar lo que llaman consciencia social, acompañada de sensibilidad y pensamiento crítico frente al entorno. En el caso de Mónica Salazar, expone que el o la profesional en psicología debe tener una perspectiva crítica pero bastante abierta a la diversidad, debido a que lo que prolifera hoy en día es la multiplicidad de aproximaciones, de intervenciones, de metodologías, de teorías, de marcos conceptuales de trabajo, y que es desde esa apertura a la diversidad que se debe posicionar críticamente para tomar decisiones frente a su realidad.

Para tener esta capacidad de pensamiento crítico, Mirta González advierte que el o la profesional en psicología debe tener un conocimiento amplio de la cultura general, los derechos humanos, las relaciones de poder, las formas de discriminación y, en general, de la sociedad en la que se inserta profesionalmente.

Por otra parte, según Mauricio Leandro se requiere adquirir destrezas que tradicionalmente han sido consideradas propias de otros campos, por ejemplo la lectura de mapas, las herramientas para poder interpretar la legislación vigente, el lenguaje de programación, la lectura de imágenes, entre muchos otros que pueden variar según el campo de inserción de la Psicología.

Henning Jensen, Mónica Salazar y Mauricio Leandro enfatizan que la Psicología es una ciencia y por lo tanto, la investigación y su difusión es fundamental. La capacidad crítica que se planteaba anteriormente no está relacionada solamente con las respuestas a los problemas, sino con estar pensando de manera constante en interrogantes y, más aun, en cómo se nos presentan los problemas. "El psicólogo es un científico ante todo, una persona que piensa en los problemas y no sólo cómo resolverlos sino si el problema está bien planteado o no" (Mauricio Leandro, comunicación personal, 18 de julio, 2012). 
Sin embargo, Mónica Salazar recuerda que tenemos una pobre cultura de divulgación de resultados de proyectos e investigaciones, y ese debería ser un aspecto fundamental a fortalecer.

$\mathrm{Al}$ respecto, según Henning Jensen, para las y los estudiantes se vuelve imprescindible el dominio de un segundo idioma y particularmente del inglés, ya que ofrece una garantía de inserción en espacios académicos internacionales, sea en eventos o publicaciones, a la vez que abre las oportunidades para los estudios de posgrado.

Otro aspecto en el que coincidieron Henning Jensen y Mauricio Leandro consiste en la capacidad de liderar procesos (tanto en equipos interdisciplinarios, en comunidades, en instituciones, etc.). Esto significa que las y los profesionales en psicología deben ser personas que, ante situaciones cotidianas o extraordinarias en sus ámbitos laborales, deben tener la capacidad de negociación, de resolución de conflictos y de ofrecer criterios fundamentados para orientar la toma de decisiones. Asimismo, deben poseer capacidad de impulsar nuevos proyectos y de visualizar nuevos ámbitos de inserción como una respuesta ante la saturación del mercado laboral, especialmente en las áreas más tradicionales. Para esto, en muchos casos es indispensable la vinculación con otras disciplinas: "la Psicología de nuestra Universidad ha sido siempre muy dinámica y muy creativa, abriendo siempre nuevos espacios y nuevos campos pero hay algunos campos que se han agotado" (Henning Jensen, comunicación personal, 19 de julio, 2012).

Estos aspectos anteriormente identificados en las entrevistas son coincidentes con los resultados de una investigación regional en la cual se identificaron como competencias requeridas por las personas, independientemente de su profesión, las siguientes: aprender a aprender, pensar y trabajar interdisciplinariamente, la digitalización, el dominio del inglés, la contextualización, la creatividad y la negociación y solución de conflictos. Asimismo, para el caso específico de Psicología se identificaron la capacidad empática, la flexibilidad cognitiva (superar dogmatismos) y la conciencia social (Rodríguez, 2013).

Finalmente, en esta amplia diversidad de competencias necesarias para la formación en Psicología, la capacidad de trabajo interdisciplinario destaca como una de las más importantes. Mirta González (comunicación personal, 2 de noviembre, 2012) afirma que a pesar de que hablamos mucho de la interdisciplinariedad, a nivel institucional muy poco se hace para fortalecerla. Los currículums son muy cerrados y sigue habiendo disputas por la división de los objetos de estudio, aun cuando la sociedad es compleja y requiere los aportes de todas las profesiones.

Las personas entrevistadas consideran que siguen vigentes las alianzas "más tradicionales" con disciplinas como la Sociología, la Antropología y la Lingüística, sin embargo, según Henning Jensen y Mauricio Leandro es necesario establecer vínculos más novedosos, por ejemplo con la Ingeniería Cognitiva, la Arquitectura, la Ingeniería Civil y la Robótica, entre otras. Incluso, se plantea que uno de los mayores retos son las carreras multidisciplinarias tales como los Estudios Urbanos. Según Mauricio Leandro, en el trabajo de la planificación urbana no sólo es necesaria la colaboración sino que es inconcebible el trabajo de la Psicología si no es de manera conjunta con Arquitectura y Derecho, por mencionar dos ejemplos.

De forma similar, Mónica Salazar argumenta sobre la importancia y la necesidad del trabajo interdisciplinario. Para ella, es necesario el desarrollo de una actitud más dialogante con otras disciplinas y perspectivas, y considera de particular relevancia para las y los profesionales en Psicología el trabajo conjunto con las áreas de ciencias sociales, ciencias de la salud y ciencias de la educación. A criterio de esta profesional, el diálogo multidisciplinario es muy provechoso ya que permite una intervención verdaderamente integral donde la persona siente que está siendo atendida por un equipo y que existe un grupo de respaldo más allá de sus redes de apoyo.

La capacidad del trabajo interdisciplinario guarda una estrecha relación con otras competencias mencionadas, tales como la capacidad de liderar procesos y la de aprovechar las herramientas y técnicas desarrolladas en otras disciplinas. Al respecto, es interesante comparar estos resultados cualitativos con los hallazgos cuantitativos obtenidos por Salazar y Prado-Calderón (2012). En dicho estudio se consultó a una muestra de 225 participantes (estudiantes, docentes, egresados/as y empleadores/as en Psicología) 
sobre los niveles de importancia y cumplimiento de 24 competencias específicas de la profesión. Estos autores encontraron que la capacidad de trabajar en equipos multi e interdisciplinarios se ubicó entre las cinco competencias más importantes para la Psicología pero además fue la cuarta competencia con el menor nivel de cumplimiento. Con dichas características, esta capacidad se ubicó como la que presentó una mayor brecha entre los niveles de importancia y los de cumplimiento, y estuvo seguida de otra competencia muy relacionada con el trabajo interdisciplinario, a saber, la capacidad de "integrar y utilizar los conocimientos de otras disciplinas".

Consideramos que estos hallazgos, derivados de dos perspectivas metodológicas muy distintas (encuesta cuantitativa y entrevistas semiestructuradas), indican de manera consistente la relevancia del trabajo interdisciplinario en el contexto actual, así como el hecho de que históricamente se ha dejado de lado en la formación de profesionales en Psicología el entrenamiento en habilidades para el trabajo coordinado con otras disciplinas. Maldonado (2011) explica que estamos en una transición paradigmática, que se inicia a partir del siglo XIX, donde la concepción de lo que compone la ciencia y el humanismo implica que las disciplinas deben compartir conocimientos para explicar fenómenos más complejos. Lo anterior, implica abrirse a otras ciencias, a otros saberes, al llamado "cruce de conocimientos, al intercambio de experiencias y al diálogo horizontal de ciencias, saberes, prácticas y disciplinas, es decir 〈abrir las ciencias sociales〉 en nuevas temáticas, instituciones y epistemologías, para comprender mejor las dinámicas sociales y humanas" (p. 177).

En el caso particular de la carrera de Psicología de la UCR, las prácticas profesionalizantes que se llevan a cabo en los cursos teórico-prácticos (módulos), así como el trabajo comunal universitario, permiten a las y los estudiantes un acercamiento interdisciplinario donde se enfrentan al trabajo conjunto con estudiantes y profesionales de gran diversidad de disciplinas. En estos espacios académicos experimentan la riqueza del intercambio de experiencias de trabajo e investigación así como nuevas formas de pensamiento. Sin embargo, a pesar de que es evidente la necesidad de una mayor formación y experiencia previa para conocer y desarrollar habilidades en la práctica del trabajo interdisciplinario, la rigidez de los planes de estudios y las directrices a nivel institucional impiden aceptar estudiantes que tiene su empadronamiento en otras unidades académicas en los llamados "cursos de carrera", lo cual se convierten en una de las principales barreras que dificultan el fortalecimiento de la interdisciplinariedad desde el proceso formativo. Si bien existen, al menos en el programa de estudios de Psicología, cursos que tienen una especificidad para estudiantes de Psicología (por ejemplo, formación en clínica), existen muchos otros, especialmente a través de los Módulos, que plantean áreas de inserción profesionalizante en donde el abordaje interdisciplinario es fundamental y vendría a enriquecer la experiencia formativa.

\section{Conclusión}

Es precisamente frente a estos escenarios sociales complejos y dinámicos que deben plantearse las interrogantes vinculadas a los retos y las complejidades que enfrenta la Psicología como disciplina y, a su vez, las Universidades Públicas para la formación de sus profesionales.

Por consiguiente, la formación de profesionales en Psicología en la UCR debe estar apegada a los propósitos de esta Universidad, en el sentido de estimular la formación de una conciencia creativa y crítica en las personas que integran la comunidad costarricense; promulgar una formación humanística con los principios de libertad, verdad, justicia, belleza, respeto a las diferencias, solidaridad, eficacia y eficiencia; contribuir al progreso de las ciencias, las artes, las humanidades y las tecnologías, reafirmando su interrelación y aplicándolas al conocimiento de la realidad costarricense y de la comunidad internacional; formar profesionales que participen eficazmente en el desarrollo del sistema de educación costarricense e impulsen y desarrollen la docencia, la investigación y la acción social; elevar el nivel cultural de la sociedad costarricense mediante la acción universitaria ${ }_{\text {s }}$ estudiar los problemas de la comunidad y participar en proyectos tendientes al pleno desarrollo de los recursos humanos, en función de un plan integral, destinado a formar un régimen social justo, que elimine las causas que 
producen la ignorancia y la miseria, así como a evitar la indebida explotación de los recursos del país (UCR, Consejo Universitario, 1974, Artículo 5).

Rita Meoño (2011), representante por el Área de Ciencias Sociales ante el Consejo Universitario de la UCR, reflexiona que la educación superior pública tiene un papel estratégico en la sociedad por sus posibilidades creativas y emancipadoras, y que esta "sigue siendo una pieza fundamental para la transformación de la realidad y la emancipación de la humanidad" (p. 3). Sin embargo, considera que en el cumplimiento de sus responsabilidades, la Universidad Pública tropieza con limitaciones y obstáculos por el momento histórico en que vivimos. Este se caracteriza por una sociedad convertida en un gran mercado donde la Universidad pasa a ser parte de la mercantilización; de un bien público (derecho social) pasa a ser un servicio. Por consiguiente, propone que "uno de los grandes desafíos hoy, es comprender críticamente el momento histórico que vivimos y sus múltiples determinantes para la educación superior en la región; para así, develarlo en aras de esbozar caminos alternativos a los impuestos” (p. 15). Asimismo, señala que uno de los grandes desafíos consiste en "impedir el sometimiento de la Universidad Pública a los intereses del capital y por el contrario vincular su quehacer a procesos de liberación política, cultural y económica, a la búsqueda de una sociedad diferente; vincularla estrechamente a la solución de problemas nacionales y/o regionales" (p. 15). Por lo tanto, resulta muy preocupante la toma de decisiones sobre las carreras que imparten las Universidades Públicas, tanto en sus sedes centrales como regionales, con base en las necesidades del mercado, olvidando el importante aporte que realizan algunas disciplinas, destacando en este artículo la Psicología en particular, en ser vigilantes y comprometidas con una sociedad justa, solidaria y respetuosa de los derechos humanos. De tal manera, queda el reto planteado para la defensa (tanto a nivel interno como externo) del modelo de Universidad Pública en que fueron inspiradas las casas de educación superior en nuestro país.

\section{Referencias}

Adell, Jordi. (1997). Tendencias en educación en la sociedad de las tecnologías de la información. EDUTEC: Revista Electrónica de Tecnología Educativa, 7, sin números de página. Recuperado de http://www.uib.es/depart/gte/revelec7.html

Arley, Alejandro. (27 de octubre de 2011). Colegio de psicólogos halla posibles delitos en exámenes de armas. La Nación, p. 20A.

Arley, Alejandro, \& Moya, Ronald. (24 de octubre de 2011). Aspirantes ganan con facilidad examen para portar armas. La Nación, p. 16A.

Asociación de Unidades Académicas de Psicología (AUAPSI). (2012). Declaración de Montevideo. Recuperado de www.psyche.unc.edu.ar/prensa/DOCUMENTO\%20MONTEVIDEO.pdf

Campos, Armando. (s. f.). Propuesta para la creación de la maestría académica en psicología comunitaria. (Informe del Programa en Posgrado en Psicología de la Universidad de Costa Rica). Recuperado de https://www.dropbox.com/s/8v32b5ogq23yw4x/Campos.pdf

Capra, Fritjof. (1992). El punto crucial: Ciencia, sociedad y cultura naciente (G. de Luis, Trad.). Buenos Aires, Argentina: Troquel. (Trabajo original publicado en 1982).

Chaves, Melissa; Ramos, Viviana, \& Sánchez, Ixa. (2013). Factores que promueven la satisfacción sexual en parejas heterosexuales del gran área metropolitana (Tesis de Licenciatura en Psicología). Universidad de Costa Rica, San José, Costa Rica.

Fernández, Evelyn. (18 de octubre de 2013). Los 10 cantones con mayor acceso a Internet en Costa Rica. El Financiero. Recuperado de http://www.elfinancierocr.com/economia-y-politica/Graficocantones-Internet-Costa-Rica_0_392960712.html

Hernández, Raquel, \& Ulloa, Irene. (2014). Condiciones laborales y su impacto sobre el proyecto de vida: Un estudio descriptivo con las personas que se graduaron de la Licenciatura en Psicología impartida en la Sede Rodrigo Facio de la Universidad de Costa Rica entre los años 2008 - 2010 (Tesis de Licenciatura en Psicología). Universidad de Costa Rica, San José, Costa Rica. 
Maldonado, Carlos. (2011). Termodinámica y complejidad: Una introducción para las ciencias sociales y humanas. Bogotá, Colombia: Desde Abajo.

Meoño, Rita. (2011). Limitaciones que afectan a la universidad pública. Ponencia presentada en el Primer Foro Institucional 2011 Universidad Pública, Sociedad y Estado de la Universidad de Costa Rica. San José, Costa Rica.

Programa Sociedad de la Información y el Conocimiento (PROSIC). (2006). Hacia la sociedad de la información y el conocimiento: Informe 2006. San José, Costa Rica: Autor.

Programa Sociedad de la Información y el Conocimiento (PROSIC). (2008). Informe 2007: Hacia la sociedad de la información y el conocimiento en Costa Rica. San José, Costa Rica: Autor.

Rodríguez, Diego. (Ed.). (2013). Educación superior en América Latina: Reflexiones y perspectivas en psicología. Bilbao, España: Universidad de Deusto.

Salazar, Zaida. (Ed.). (2009). Caracterización de la Escuela de Psicología Universidad de Costa Rica: Experiencia de la autoevaluación y la reacreditación. San José, Costa Rica: Escuela de Psicología de la Universidad de Costa Rica.

Salazar, Zaida, \& Prado-Calderón, Jorge Esteban. (2012). Valoración de competencias específicas del profesional en psicología desde la Universidad de Costa Rica. Revista Costarricense de Psicología, 31(1-2), 40-63.

Santos, Boaventura de Sousa. (2005). La universidad en el siglo XXI: Para una reforma democrática y emancipadora de la universidad. México D. F., México: Universidad Nacional Autónoma de México.

Universidad de Costa Rica, Consejo Universitario. (1974). Estatuto Orgánico de la Universidad de Costa Rica. San José, Costa Rica: Autor.

\section{Entrevistas}

Dr. Mauricio Leandro Rojas. Docente asociado de la Escuela de Psicología e investigador de la Escuela de Psicología y el Instituto de Investigaciones Psicológicas, UCR. 18 de julio de 2012, San José, Costa Rica.

Dr. Henning Jensen Pennington. Catedrático de la Escuela de Psicología de la UCR, actualmente Rector de la UCR. 19 de julio de 2012, San José, Costa Rica.

Dra. Mirta González Suárez. Docente catedrática e investigadora de la Escuela de Psicología de la UCR. 2 de noviembre de 2012, San José, Costa Rica.

Dra. Mónica Salazar Villanea. Docente adjunta de la Escuela de Psicología de la UCR e investigadora del Instituto de Investigaciones Psicológicas. 24 de enero de 2013, San José, Costa Rica. 
